\title{
Management of singlet and triplet excitons for efficient white organic light-emitting devices
}

\author{
Yiru Sun ${ }^{1}$, Noel C. Giebink ${ }^{1}$, Hiroshi Kanno ${ }^{1}$, Biwu Ma ${ }^{2}$, Mark E. Thompson ${ }^{2} \&$ Stephen R. Forrest ${ }^{1} \dagger$
}

Lighting accounts for approximately 22 per cent of the electricity consumed in buildings in the United States, with 40 per cent of that amount consumed by inefficient $\left(\sim 15 \mathrm{~lm} \mathrm{~W}^{-1}\right)$ incandescent lamps ${ }^{1,2}$. This has generated increased interest in the use of white electroluminescent organic light-emitting devices, owing to their potential for significantly improved efficiency over incandescent sources combined with low-cost, high-throughput manufacturability. The most impressive characteristics of such devices reported to date have been achieved in all-phosphor-doped devices, which have the potential for 100 per cent internal quantum efficiency ${ }^{2}$ : the phosphorescent molecules harness the triplet excitons that constitute three-quarters of the bound electron-hole pairs that form during charge injection, and which (unlike the remaining singlet excitons) would otherwise recombine non-radiatively. Here we introduce a different device concept that exploits a blue fluorescent molecule in exchange for a phosphorescent dopant, in combination with green and red phosphor dopants, to yield high power efficiency and stable colour balance, while maintaining the potential for unity internal quantum efficiency. Two distinct modes of energy transfer within this device serve to channel nearly all of the triplet energy to the phosphorescent dopants, retaining the singlet energy exclusively on the blue fluorescent dopant. Additionally, eliminating the exchange energy loss to the blue fluorophore allows for roughly 20 per cent increased power efficiency compared to a fully phosphorescent device. Our device challenges incandescent sources by exhibiting total external quantum and power efficiencies that peak at $18.7 \pm 0.5$ per cent and $37.6 \pm 0.6 \mathrm{~lm} \mathrm{~W} \mathrm{~W}^{-1}$, respectively, decreasing to $18.4 \pm 0.5$ per cent and $23.8 \pm 0.5 \mathrm{~lm} \mathrm{~W} \mathrm{~W}^{-1}$ at a high luminance of $500 \mathrm{~cd} \mathrm{~m}^{-2}$.

Electrophosphorescent organic light-emitting devices (OLEDs) have been shown to harvest $100 \%$ of the excitons generated by electrical injection, corresponding to a fourfold increase in efficiency compared to that achievable in singlet-harvesting fluorescent OLEDs $^{3}$. In this context, electrophosphorescent white OLEDs (WOLEDS) have been reported to exhibit ${ }^{4-7}$ high quantum (5$12 \%)$ and luminous power efficiencies $\left(6-20 \mathrm{~lm} \mathrm{~W}^{-1}\right)$ at brightnesses $<100 \mathrm{~cd} \mathrm{~m}^{-2}$. To date, however, blue electrophosphorescent devices have exhibited short operational lifetimes ${ }^{8}$ that limit the colour stability of all-phosphor-doped WOLEDs. Also, compared with their fluorescent counterparts, WOLEDs employing phosphorescent blue dopants excited via the conductive host introduce an approximately $0.8 \mathrm{eV}$ exchange energy loss in power efficiency. This results from the energetic relaxation following intersystem crossing into the emissive triplet state. This loss can be avoided by resonant injection from the hole transport layer (HTL) and electron transport layer (ETL) into the phosphor triplet state ${ }^{6,9}$, but the subsequent transfer to green and red dopants required to generate white light can reintroduce these parasitic energy losses. Here we demonstrate a new WOLED architecture that uses a fluorescent emitting dopant to harness all electrically generated high energy singlet excitons for blue emission, and phosphorescent dopants to harvest the remainder of lower-energy triplet excitons for green and red emission.

This structure takes advantage of the fortuitous connection between the proportion of singlets dictated by spin statistics (that is, one singlet versus three triplets are produced by electrical excitation $^{10}$ ) and the roughly $25 \%$ contribution of blue to the perceived white light spectrum. This allows for resonant energy transfer from both the host singlet and triplet energy levels that minimizes exchange energy losses, thereby maximizing device power efficiency while maintaining the potential for unity internal quantum efficiency (IQE). This approach has the further advantages of a stable white balance with current, a high efficiency at high brightness due to reduced geminate exciton recombination ${ }^{11}$, and an enhanced lifetime due to the combined use of a stable fluorescent blue, and long lived phosphorescent green and red, dopants in a single emissive region.

The WOLED consists of a blue fluorophore, 4,4'-bis(9-ethyl-3carbazovinylene)-1,1'-biphenyl $(\mathrm{BCzVBi})^{12}$, doped in a region spatially separate from the highly efficient green and red phosphorescent dopants fac-tris(2-phenylpyridine) iridium $\left(\operatorname{Ir}(\mathrm{ppy})_{3}\right)$ and iridium(III) bis(2-phenyl quinolyl-N, $\mathrm{C}^{2^{\prime}}$ ) acetylacetonate (PQIr), respectively. All lumophores are doped into a single, common conductive host, 4,4'-bis(N-carbazolyl)biphenyl (CBP), to form the extended emissive layer (EML) which is sandwiched between the electron transporting/hole blocking layer of 2,9-dimethyl-4,7diphenyl-1,10-phenanthroline (BCP), and the 4,4'-bis[N-(1naphthyl)-N-phenyl-amino]-biphenyl ( $\alpha$-NPD) HTL.

The principle of device operation is illustrated in Fig. 1. Excitons are formed on the host with a singlet-to-triplet formation ratio of $\chi_{\mathrm{s}} / \chi_{\mathrm{t}}$. Singlet excitons are transferred following a resonant Förster process onto the lightly doped (5\%) blue fluorophore as opposed to direct trap formation ${ }^{12}$. The non-radiative host triplets, however, cannot efficiently transfer to the fluorophore by the Förster mechanism, or by Dexter transfer owing to the low doping concentration. On the other hand, triplets typically have long diffusion lengths ${ }^{10}$ $(\sim 100 \mathrm{~nm})$, and hence can migrate into the centre of the EML where they transfer onto the phosphors. Resonant transfer of the host triplet onto the green phosphor avoids exchange energy losses at this stage, although there are some unavoidable losses in transferring into the lowest energy red phosphor. Finally, placing an undoped host spacer with a thickness larger than the Förster radius $(\sim 3 \mathrm{~nm})$ between the blue fluorophore and the phosphors prevents direct energy transfer from the blue dopant to the green and red phosphors. This device architecture is unique in that the singlet and triplet excitons are harvested along completely independent channels, and hence the transfer from host to dopant for both species can be separately

${ }^{1}$ Department of Electrical Engineering, Princeton Institute for the Science and Technology of Materials (PRISM), Princeton University, Princeton, New Jersey 08544, USA.

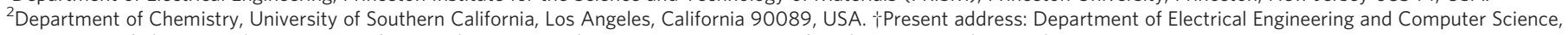
Department of Physics, and Department of Materials Science and Engineering, University of Michigan, Ann Arbor, Michigan 48109, USA. 
optimized to be nearly resonant, thereby minimizing energy losses while maintaining a unity IQE.

Figure 2 provides evidence for this transfer mechanism by comparing the un-normalized electroluminescent spectra of three devices at a current density of $J=100 \mathrm{~mA} \mathrm{~cm}^{-2}$. Device I has a 16-nm-thick CBP spacer placed between the two 5-nm-thick $5 \mathrm{wt} \% \mathrm{BCzVBi}: \mathrm{CBP}$ layers at each side of the EML, whereas device II has a 25-nm-thick uniformly doped $5 \mathrm{wt} \% \mathrm{BCzVBi}$ :CBP EML. Both devices have nearly identical emission spectra and external quantum efficiencies (EQE) of $\eta_{\text {ext }}=(2.6 \pm 0.2) \%$, indicating that ostensibly $100 \%$ of the exciton formation occurs at the edges of the EML. Furthermore, lack of short wavelength CBP emission in device I suggests that the charge density in the middle region of EML available for exciton formation directly on the host is negligible. When a 20 -nm-thick $3 \mathrm{wt} \% \operatorname{Ir}(\mathrm{ppy})_{3}: \mathrm{CBP}$ layer is inserted in the EML and separated from the two $5 \mathrm{wt} \%$ BCzVBi:CBP regions by 4 -nm-thick undoped CBP spacers (device III), the total efficiency is increased to $\eta_{\text {ext }}=(5.2 \pm 0.2) \%$, with the additional $2.6 \%$ emission coming from $\operatorname{Ir}(\mathrm{ppy})_{3}$. From this we conclude that exciton diffusion from the point of origin at the edges of the EML, rather than direct charge trapping and exciton formation on the phosphor, dominates, because carriers trapped by $\operatorname{Ir}(\mathrm{ppy})_{3}$ would result in a noticeable decrease in the $\mathrm{BCzVBi}$ emission. Triplet diffusion from the edges of the EML to the phosphorescent doped region is consistent with previous observations in red fluorescent/phosphorescent OLEDs, where the fluorophore 'filters' out the singlet excitons, leaving only triplets to diffuse to a spatially separated phosphor doped region ${ }^{3}$.

To determine whether the location of the exciton formation region is predominantly at either the HTL/EML or EML/ETL interface, we compared the emission from two comparable devices with opposite symmetries, where the structure consisted of either NPD $(30 \mathrm{~nm}) / 5 \mathrm{wt} \% \mathrm{BCzVBi}: \mathrm{CBP}(10 \mathrm{~nm}) / \mathrm{CBP}(20 \mathrm{~nm}) / \mathrm{BCP}(40 \mathrm{~nm})$, or NPD $(30 \mathrm{~nm}) / \mathrm{CBP}(20 \mathrm{~nm}) / 5 \mathrm{wt} \%$ BCzVBi:CBP: $(10 \mathrm{~nm}) / \mathrm{BCP}$ $(40 \mathrm{~nm})$. The corresponding maximum efficiencies of these devices

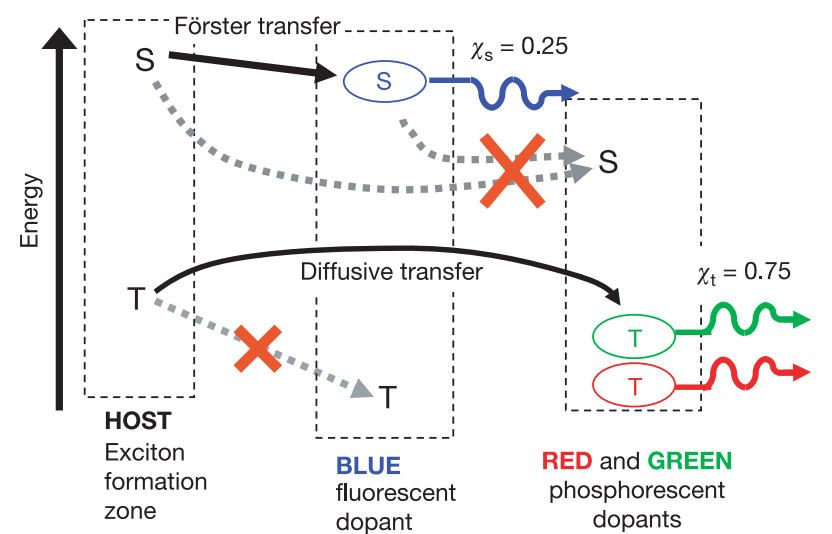

Figure 1 Proposed energy transfer mechanisms in the fluorescent/ phosphorescent WOLED. This illustrates the separate channels for triplet (T) and singlet (S) formation and transfer directly onto their corresponding emissive dopants. The majority of excitons are formed in the host material with a singlet-to-triplet formation ratio of $\chi_{\mathrm{s}} / \chi_{\mathrm{t}}$. The singlet excitons in the two formation regions at each side of the light emitting layer (EML) are rapidly, and near-resonantly, transferred to the blue fluorescent dye located in these regions. The phosphor-doped region is located in the centre of the EML and separated from the exciton formation zones by spacers of undoped host material. The triplets then diffuse efficiently to the central region, where they transfer to the lower energy green or red phosphor dopants, again by a nearly resonant process to the green dopant triplet manifold, and with some energy loss to the red triplet. Diffusion of singlet excitons to the phosphor dopants is negligible due to their intrinsically short diffusion lengths ${ }^{23}$. Parasitic effects of charge trapping onto the phosphorescent dopants are discussed in the text. were $\eta_{\text {ext }}=(1.4 \pm 0.1) \%$ and $(1.8 \pm 0.1) \%$, both smaller than $\eta_{\text {ext }}=(2.6 \pm 0.2) \%$ for device II. Moreover, CBP emission at a peak wavelength of $\lambda=390 \mathrm{~nm}$ is observed in the first device, and $\alpha$-NPD emission at $\lambda=430 \mathrm{~nm}$ is observed in the second structure. These observations suggest that excitons are generated at both the HTL/EML and EML/ETL interfaces, consistent with the ambipolar conductivity of $\mathrm{CBP}^{13,14}$. Exciton formation at the edges, with correspondingly low generation in the bulk of the EML, can be understood as follows: large densities of holes $(p)$ and electrons $(n)$ pile up at the energy barriers at two EML interfaces. The exciton formation probability, which is $\sim n \times p$, is thus also significantly higher at these locations as compared with the EML bulk.

On the basis of these results, WOLEDs were fabricated by doping the middle region of the EML with both the green and red phosphorescent dopants (Fig. 3a, inset). As the high energy of the highest occupied molecular orbital of PQIr suggests that it can trap holes in the CBP host, a slight decrease of the blue emission intensity is observed in the WOLED. Fitting of the WOLED spectrum in Fig. 3b with the individual dopant spectra suggests that the ratio of emission from fluorescent to phosphorescent dopants approaches the ratio of $1 / 3$, consistent with the singlet-to-triplet exciton formation ratio in emissive organic materials ${ }^{10,15,16}$. Furthermore, given the performance characteristics of the purely fluorescent $\mathrm{BCzVBi}$ device (device II in Fig. 2), we also find that the fraction of excitons trapped by, and formed directly on, the phosphorescent dopants in the EML is $\chi_{\text {trap }}=(18 \pm 5) \%$ (see Methods). That is, approximately $20 \%$ of the excitons are formed by direct trapping on the phosphor dopants, whereas the remaining $80 \%$ are formed at the edges of the EML, at which point the triplets subsequently diffuse into the centre where they are transferred from host to phosphor dopant before emission.

A maximum forward viewing EQE of $\eta_{\text {ext }}=(11.0 \pm 0.3) \%$ is achieved at a current density $J=(1.0 \pm 0.6) \mathrm{mA} \mathrm{cm}^{-2}$, and decreases only slightly to $\eta_{\text {ext }}=(10.8 \pm 0.3) \%$ at a high forward viewing luminance of $500 \mathrm{~cd} \mathrm{~m}^{-2}$ (Fig. 3a). This device gives a maximum forward viewing power efficiency of $\eta_{\mathrm{p}}=(22.1 \pm 0.3) \mathrm{lm} \mathrm{W}^{-1}$. As illumination sources are generally characterized by their total

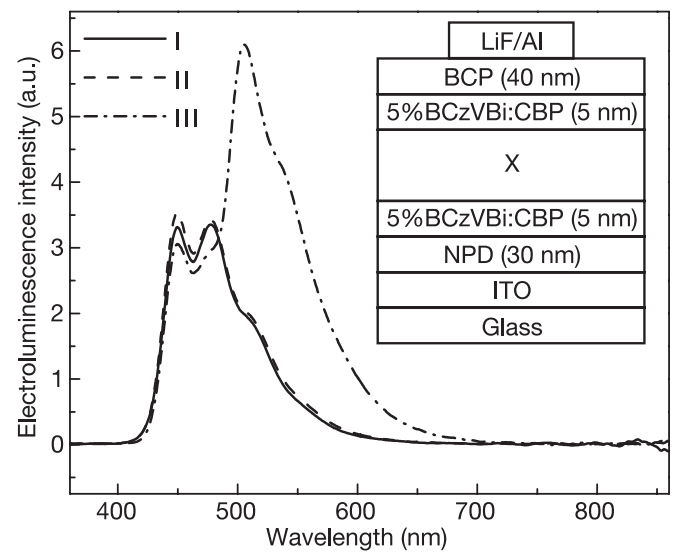

Figure 2 | Un-normalized electroluminescence spectra of three device structures shown in the inset. The spectra were measured at a current density of $100 \mathrm{~mA} \mathrm{~cm}^{-2}$. Inset, schematic cross-section of the device; see text for definitions of abbreviations used. $X=C B P(16 \mathrm{~nm})$ for device I; $\mathrm{X}=5 \mathrm{wt} \% \mathrm{BCzVBi}: \mathrm{CBP}(15 \mathrm{~nm})$ for device II; $\mathrm{X}=\mathrm{CBP}(4 \mathrm{~nm}) / 3 \mathrm{wt} \%$ $\operatorname{Ir}(\text { ppy })_{3}:$ CBP $(20 \mathrm{~nm}) / C B P(4 \mathrm{~nm})$ for device III. Quantitative comparison of the three spectra suggests that excitons are primarily formed at the two interfaces, and that fluorescent doping across the entire emission layer does not increase the blue luminescence intensity (compare devices I and II). However, doping the middle of the EML with the phosphor $\operatorname{Ir}(p p y)_{3}$ results in additional green emission without a corresponding decrease in blue, $\mathrm{BCzVBi}$ emission (devices I and III), indicating that triplets formed on CBP are efficiently transferred to $\operatorname{Ir}(\mathrm{ppy})_{3}$, whereas the $\mathrm{BCzVBi}$ acts to harvest, or 'filter out', all of the singlets. 
emitted power, this device therefore has maximum total efficiencies ${ }^{6}$ of $\eta_{\mathrm{p}, \mathrm{t}}=(37.6 \pm 0.6) \mathrm{lm} \mathrm{W}^{-1}$, and $\eta_{\text {ext }, \mathrm{t}}=(18.7 \pm 0.5) \%$. At a practical surface luminance of $500 \mathrm{~cd} \mathrm{~m}^{-2}, \eta_{\mathrm{p}, \mathrm{t}}=(23.8 \pm 0.5)$ $\operatorname{lm~} \mathrm{W}^{-1}$, or approximately $50 \%$ greater than for common incandescent lighting. Although the commercially available blue fluorophore has a low $\eta_{\text {ext }}=2.7 \%$ (compared with a maximum expected $5 \%$ achieved in the literature), the WOLED performance, nevertheless, represents a considerable improvement over the best all-phosphorescent devices previously reported ${ }^{6,7,17}$ (see Supplementary Information).

The intrinsic singlet-to-triplet ratio and the separation of the
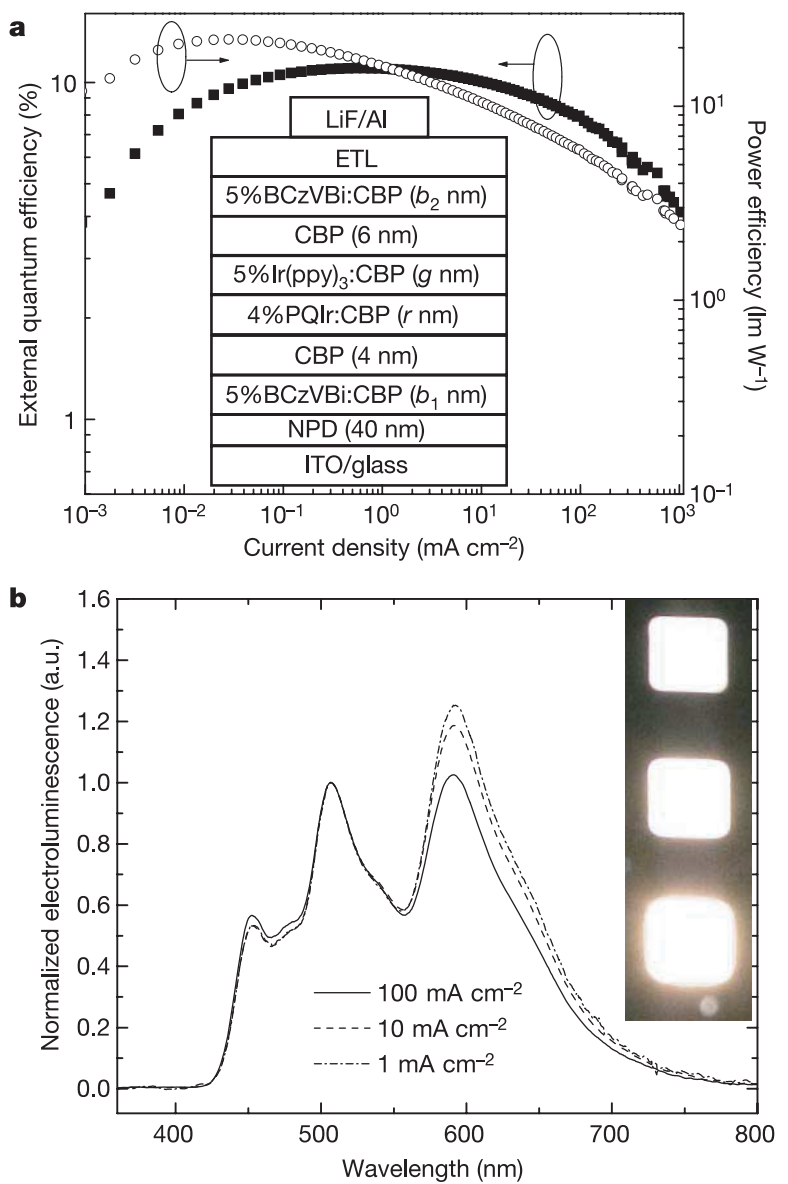

Figure 3 | Performance characteristics of the fluorescent/phosphorescent WOLED. a, Forward viewing external quantum efficiency (filled squares) and power efficiency (open circles) versus current density of the WOLED shown in the inset. The forward viewing external quantum efficiency peaks at $\eta_{\mathrm{ext}}=(11.0 \pm 0.3) \%$ at $J=(1.0 \pm 0.6) \mathrm{mA} \mathrm{cm}^{-2}$, and decreases slightly to $\eta_{\mathrm{ext}}=(10.8 \pm 0.3) \%$ at a forward viewing luminance of $500 \mathrm{~cd} \mathrm{~m}^{-2}$. The maximum forward viewing power efficiency is $\eta_{\mathrm{p}}=(22.1 \pm 0.3) \mathrm{lm} \mathrm{W}^{-1}$, with total peak and high luminance efficiencies of $\eta_{\mathrm{p}, \mathrm{t}}=(37.6 \pm 0.6)$ and $(23.8 \pm 0.5) \mathrm{lm} \mathrm{W}^{-1}$ at $500 \mathrm{~cd} \mathrm{~m}^{-2}$, respectively. The forward viewing luminance at $1 \mathrm{~A} \mathrm{~cm}^{-2}$ is $(83,000 \pm 7,000) \mathrm{cd} \mathrm{m}^{-2}$. The drive voltage for this device is $(6.0 \pm 0.5) \mathrm{V}$ at $J=10 \mathrm{~mA} \mathrm{~cm}^{-2}$. Inset, schematic structure of the WOLED with the following layer thicknesses: $b_{1}=15 \mathrm{~nm}, b_{2}=10 \mathrm{~nm}$, $r=8 \mathrm{~nm}, g=12 \mathrm{~nm}$, and with an electron transport layer (ETL) consisting of 20-nm 4,7-diphenyl-1,10-phenanthroline (BPhen) followed by $20-\mathrm{nm} \mathrm{Li}$ doped BPhen in 1:1 molar ratio. Here, $\mathrm{BPhen}$ is used to further reduce device drive voltage. When $40-\mathrm{nm} \mathrm{BCP} \mathrm{is} \mathrm{used} \mathrm{as} \mathrm{ETL} \mathrm{and} b_{1}=10 \mathrm{~nm}$,

$b_{2}=10 \mathrm{~nm}, r=12 \mathrm{~nm}$ and $g=8 \mathrm{~nm}, \eta_{\text {ext }}$ and CRI are nearly identical to the above structure. $\mathbf{b}$, Normalized electroluminescence spectra of WOLED emission at various current densities. Note that colour dependence on current density is minimal, with $\mathrm{CRI}=85$ at all three values of current density. Inset, images of three, $4.5 \mathrm{~mm}^{2}$ devices, each driven at four times the drive current (from 1.7 to $28 \mathrm{~mA} \mathrm{~cm}^{-2}$ ) of the device above it in the array (equivalent to a two f-stop difference in illumination) to show the colour stability of the emission. channels in harvesting the two excitonic species gives a well-balanced and largely current-independent colour rendition, resulting in a colour rendering index of CRI $=85$ at all current densities studied, which is the highest CRI among the reported values for a WOLED. The Commission Internationale d'Eclairage (CIE) coordinates have a negligible shift from $(0.40,0.41)$ at $1 \mathrm{~mA} \mathrm{~cm}^{-2}$ to $(0.38,0.40)$ at $100 \mathrm{~mA} \mathrm{~cm}^{-2}$. This differs from observations of an all-phosphordoped WOLED, where blue emission becomes stronger with increasing driving voltage ${ }^{6}$ owing to the requirement for high energy excitation of the blue phosphor. In the inset of Fig. $3 \mathrm{~b}$ are images of three devices, each driven at 4 times higher drive current than the device above it in the array, to show the colour stability of the emission.

To further understand exciton diffusion, in Fig. 4a we plot (open circles) $\eta_{\text {ext }}$ due to $\operatorname{Ir}(\text { ppy })_{3}$ emission versus the position $(x)$ of a thin $(5 \mathrm{~nm})$ slab of $5 \mathrm{wt} \% \operatorname{Ir}(\mathrm{ppy})_{3}: \mathrm{CBP}$ located at various distances from
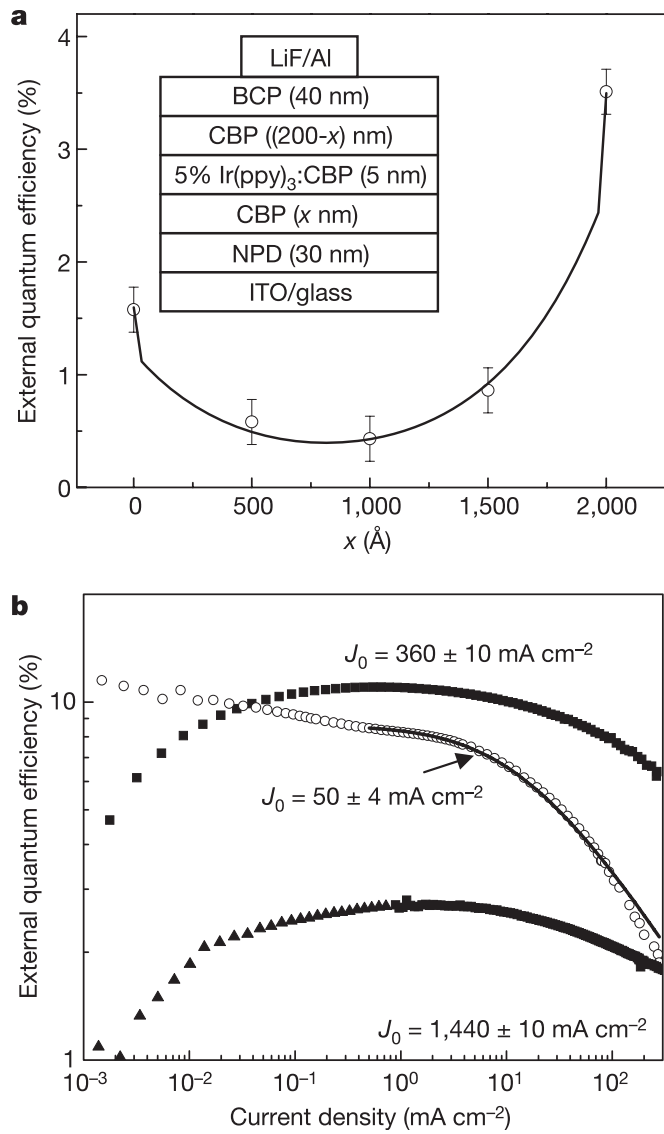

Figure 4 | Triplet diffusion profile and reduced efficiency roll-off at high currents. a, External quantum efficiency (open circles) from $\operatorname{Ir}(\mathrm{ppy})_{3}$ emission at $10 \mathrm{~mA} \mathrm{~cm}^{-2}$ versus distance between the $50-\AA$ slab of $5 \mathrm{wt} \%$ $\operatorname{Ir}(\text { ppy) })_{3}: \mathrm{CBP}$ and the NPD/CBP interface in the structure shown inset. A fit following equation (2) for triplet diffusion gives the solid curve and a triplet diffusion length of $L_{\mathrm{D}}=(460 \pm 30) \AA$. The error bars indicate the standard deviations in measurement. Inset, schematic cross-section of the test structures: NPD $(30 \mathrm{~nm}) / \mathrm{CBP}(x \mathrm{~nm}) / 5 \mathrm{wt} \% \operatorname{Ir}(\mathrm{ppy})_{3}: \mathrm{CBP}(5 \mathrm{~nm}) / \mathrm{CBP}$ $((200-x) \mathrm{nm}) / \mathrm{BCP}(40 \mathrm{~nm})$, with $x=0,50,100,150,200 . \mathrm{b}$, Comparison of external quantum efficiency roll-off. Open circles depict the performance of the all-phosphor white device of ref. 6, in comparison to the white device of this work (squares), and a blue fluorescent BCzVBi device (triangles). The high current roll-off of the phosphor device is described by triplet-triplet annihilation (fit shown as solid line), yielding an onset current density $J_{0}=(50 \pm 4) \mathrm{mA} \mathrm{cm}^{-2}$. The device of this work clearly demonstrates a roll-off that appears qualitatively similar to that of the all-fluorescent device. For comparison, $J_{0}=(360 \pm 10) \mathrm{mA} \mathrm{cm}^{-2}$ and $J_{0}=(1,440 \pm 10) \mathrm{mA} \mathrm{cm}^{-2}$ for the WOLED of this work and the all-fluorescent device, respectively. 
the HTL/EML interface within a 200-nm-thick CBP EML (see Fig. 4a inset). Fitting (solid curve, see Methods) of the efficiency versus $x$ yields a triplet diffusion length of $L_{\mathrm{D}}=(460 \pm 30) \AA$, and predicts that $(75 \pm 5) \%$ of the phosphorescent emission results from triplet exciton diffusion from the adjacent EML interfaces, in agreement with the value calculated from analysis of the spectral content of the emission.

Compared with previous all-phosphor, high efficiency WOLEDs, the device also has a less pronounced efficiency roll-off at high current densities. For example, in Fig. $4 \mathrm{~b}$ we show a comparison of $\eta_{\text {ext }}$ versus $J$ for an all-phosphor white device ${ }^{6}$, the device of this work, and a fluorescent BCzVBi device II. The high-current decline in $\eta_{\text {ext }}$ of the all-phosphor white is due to triplet-triplet annihilation ${ }^{14,18}$. In contrast, there is a striking resemblance between the efficiency roll-off of the current device, and that of device II. Modelling of the roll-offs in these two structures is complicated by recombination processes such as exciton-polaron quenching ${ }^{11}$, singlet-triplet annihilation, and field-induced exciton dissociation. Nevertheless, for both of these latter devices, the current density at the point where $\eta_{\text {ext }}$ has declined by half from its peak is $>7$ times that of the conventional phosphor device, while the peak EQE occurs at a value of $J$ nearly 1,000 times larger. The apparent absence of triplet-triplet annihilation suggests that the highest density of triplet excitons is at the interfaces in the fluorescent doped regions, where they subsequently diffuse towards the centre, thereby lowering the local density (Fig. 4a) in the region of the guest phosphors. The reduced sensitivity of $\eta_{\text {ext }}$ to current density is another clear difference between the WOLED of this study and previous, high efficiency all-phosphor devices.

We note that the efficiency of the present device can be further improved by using fluorescent dopants ${ }^{19}$ with IQE $=25 \%$ and phosphors $^{20,21}$ with IQE $=100 \%$, resulting in a total WOLED internal quantum efficiency of $100 \%$. Using such 'ideal' chromophores, whose spectra are the same as the current dopants used, an approximately $34 \%$ total EQE and $60 \mathrm{~lm} \mathrm{~W}^{-1}$ power efficiency can in principle be achieved using this structure, corresponding to a fourfold increase over incandescent power efficiency, and even competing with high efficiency, high CRI fluorescent lighting sources. As noted above, the exchange energy difference between the host singlet and dopant triplet states can lead to a loss of luminance efficiency in allphosphor doped WOLEDs. By applying this design concept to systems where the host singlet is resonant with the blue fluorophore singlet state, and the host triplet is resonant with the green phosphor triplet level, this structure could have a power efficiency improvement of $\sim 20 \%$ compared to similarly ideal all-phosphor devices. The highly efficient WOLED structure reported here, with a colour rendition that is unusually independent of current density, has potential for use in the next generation of sources for solid-state indoor lighting.

\section{METHODS}

Device manufacture. Devices were grown on clean glass substrates pre-coated with a 150-nm-thick layer of indium tin oxide (ITO) with a sheet resistance of $20 \Omega$ per square. All organic layers were grown in succession without breaking vacuum $\left(\sim 10^{-7}\right.$ torr $)$. After organic film deposition, a shadow mask with 1-mm-diameter openings was affixed in a $\mathrm{N}_{2}$ filled glove box before the cathode (consisting of 8 - $\AA$-thick LiF), followed by a $500-\AA$-thick $\mathrm{Al}$ cap, was deposited by high vacuum $\left(10^{-6}\right.$ torr $)$ thermal evaporation. Current-voltage and EQE measurements were carried out using a semiconductor parameter analyser (HP 4145) and a calibrated Si photodiode (Hamamatsu S3584-08) following standard procedures ${ }^{22}$.

Data analysis. To interpret the emission spectrum, the WOLED EQE is expressed as:

$$
\eta_{\text {ext }}=\left(1-\chi_{\text {trap }}\right) \eta_{\mathrm{B}}+\left[\left(1-\chi_{\text {trap }}\right) \chi_{\mathrm{t}}+\chi_{\text {trap }}\right] \eta_{\mathrm{GR}}
$$

where $\eta_{\mathrm{B}}$ and $\eta_{\mathrm{GR}}$ are respectively the EQEs of a singly doped blue fluorescent device and the comparable singly doped green and red phosphorescent devices, and $\chi_{\text {trap }}$ is the fraction of excitons trapped and formed directly on the phosphorescent dopants in the EML. By fitting the WOLED spectrum in
Fig. $3 \mathrm{~b}$ at $J=100 \mathrm{~mA} \mathrm{~cm}^{-2}$ with the electroluminescence spectra of the three individual dopant materials, and accounting for photon energy in these power spectra, we find that $(20 \pm 2) \%$ of the total quantum efficiency is due to emission from the blue fluorescent dopant, and $(80 \pm 2) \%$ is from green and red phosphorescent dopants. Given the performance characteristics $\left(\eta_{\mathrm{B}}\right)$ of the purely BCzVBi device (device II in Fig. 2), we calculate $\chi_{\text {trap }}=(18 \pm 5) \%$ from the first term in equation (1).

Modelling. Exciton diffusion through the EML is modelled as shown in Fig. 4a (solid line) as follows: in steady state, and assuming that all singlet formation occurs at the HTL/EML $(x=0)$, and EML/ETL interfaces $(x=200 \mathrm{~nm})$ (in Fig. 4a), a solution to the triplet diffusion equation gives:

$$
\begin{aligned}
n(x)= & \frac{1}{\sinh \left(\frac{L}{L_{\mathrm{D}}}\right)}\left[\chi_{\mathrm{t}} n_{\mathrm{R}} \sinh \left(\frac{x}{L_{\mathrm{D}}}\right)+\chi_{\mathrm{t}} n_{\mathrm{L}} \sinh \left(\frac{L-x}{L_{\mathrm{D}}}\right)\right] \\
& +\chi_{\mathrm{s}} n_{\mathrm{L}} \delta(x)+\chi_{\mathrm{s}} n_{\mathrm{R}} \delta(x-L)
\end{aligned}
$$

where $n$ is the total exciton density: $n(x=0)=n_{\mathrm{L}}$ and $n(x=L=200 \mathrm{~nm})=$ $x=L=200 \mathrm{~nm})=n_{\mathrm{R}}, L_{\mathrm{D}}$ is the triplet diffusion length, and the delta function terms account for the presence of contributing singlets at the interfaces. As the $\mathrm{EQE}$ from $\operatorname{Ir}(\mathrm{ppy})_{3}$ emission is proportional to the exciton density in the $\operatorname{Ir}(\text { ppy) })_{3}$-doped slab, Fig. 4a (solid curve) shows the fit of the efficiency at $J=10 \mathrm{~mA} \mathrm{~cm}^{-2}$ versus $x$ using equation (2) and $\chi_{\mathrm{t}}=3 \chi_{\mathrm{s}}$, from which we infer $L_{\mathrm{D}}=(460 \pm 30) \AA$ (error bars account for the spread in fits at additional current densities). With this calculated diffusion length, integration of the total exciton density in the phosphorescent doped region in the WOLED predicts that $(75 \pm 5) \%$ of the phosphorescent emission results from triplet exciton diffusion from the adjacent EML interfaces, in agreement with the value calculated from analysis of the spectral content of the emission (equation (1)).

\section{Received 19 June 2005; accepted 13 February 2006.}

1. US Department of Energy National Lighting Inventory and Energy Consumption Estimate Vol. 1, xii (US Govt Printing Office, Washington DC, 2001).

2. D'Andrade, B. W. \& Forrest, S. R. White organic light-emitting devices for solid-state lighting. Adv. Mater. 16, 1585-1595 (2004).

3. Baldo, M. A. et al. Highly efficient phosphorescent emission from organic electroluminescent devices. Nature 395, 151-154 (1998).

4. Adamovich, V. et al. High efficiency single dopant white electrophosphorescent light emitting diodes. N. J. Chem. 26, 1171-1178 (2002).

5. D'Andrade, B. W. \& Forrest, S. R. Effects of exciton and charge confinement on the performance of white organic $p$-i-n electrophosphorescent emissive excimer devices. J. Appl. Phys. 94, 3101-3109 (2003).

6. D'Andrade, B. W., Holmes, R. J. \& Forrest, S. R. Efficient organic electrophosphorescent white-light-emitting device with a triple doped emissive layer. Adv. Mater. 16, 624-627 (2004).

7. Tokito, S., lijima, T., Tsuzuki, T. \& Sato, F. High-efficiency white phosphorescent organic light-emitting devices with greenish-blue and red-emitting layers. Appl. Phys. Lett. 83, 2459-2461 (2003).

8. Tung, Y. J. et al. A high efficiency phosphorescent white OLED for LCD backlight and display applications. Proc. Soc. Inform. Display 35, 48-51 (2004)

9. Holmes, R. J. et al. Efficient, deep-blue organic electrophosphorescence by guest charge trapping. Appl. Phys. Lett. 83, 3818-3820 (2003).

10. Baldo, M. A. Excitonic singlet-triplet ratio in a semiconducting organic thin film. Phys. Rev. B 66, 14422-14428 (1999).

11. Kalinowski, J. et al. Triplet energy exchange between fluorescent and phosphorescent organic molecules in a solid state matrix. Chem. Phys. 297, 39-48 (2004).

12. Hosokawa, C., Higashi, H., Nakamura, H. \& Kusumoto, T. Highly efficient blue electroluminescence from a distyrylarylene emitting layer with a new dopant. Appl. Phys. Lett. 67, 3853-3855 (1995).

13. Adachi, C., Thompson, M. E. \& Forrest, S. R. Architectures for efficient electrophosphorescent organic light-emitting devices. IEEE J. Select. Top. Quant. Electron. 8, 372-377 (2002).

14. Baldo, M. A. \& Forrest, S. R. Transient analysis of organic electrophosphorescence: I. Transient analysis of triplet energy transfer. Phys. Rev. B 62, 10958-10966 (2000).

15. Reufer, $M$. et al. Spin-conserving carrier recombination in conjugated polymers. Nature Mater. 4, 340-346 (2005).

16. Segal, M., Baldo, M. A., Holmes, R. J., Forrest, S. R. \& Soos, Z. G. Excitonic singlet-triplet ratios in molecular and polymeric organic materials. Phys. Rev. $B$ 68, 075211 (2003).

17. Qin, D. S. \& Tao, Y. White organic light-emitting diode comprising of blue fluorescence and red phosphorescence. Appl. Phys. Lett. 86, 113507 (2005).

18. Baldo, M. A., Adachi, C. \& Forrest, S. R. Transient analysis of organic electrophosphorescence. II. Transient analysis of triplet-triplet annihilation. Phys. Rev. B 62, 10967-10977 (2000).

19. Murata, H., Kafafi, Z. H. \& Uchida, M. Efficient organic light-emitting diodes 
with undoped active layers based on silole derivatives. Appl. Phys. Lett. 80, 189-191 (2002).

20. Kawamura, Y. et al. $100 \%$ phosphorescence quantum efficiency of $\operatorname{Ir}(\mathrm{III})$ complexes in organic semiconductor films. Appl. Phys. Lett. 86, 071104 (2005).

21. Adachi, C., Baldo, M. A., Thompson, M. E. \& Forrest, S. R. Nearly $100 \%$ internal phosphorescence efficiency in an organic light emitting device. J. Appl. Phys. 90, 5048-5051 (2001).

22. Forrest, S. R., Bradley, D. D. C. \& Thompson, M. E. Measuring the efficiency of organic light-emitting devices. Adv. Mater. 15, 1043-1048 (2003)

23. Sokolik, I., Priestley, R., Walser, A. D., Dorsinville, R. \& Tang, C. W. Bimolecula reactions of singlet excitons in tris(8-hydroxyquinoline) aluminum. Appl. Phys. Lett. 69, 4168-4170 (1996)
Supplementary Information is linked to the online version of the paper at www.nature.com/nature.

Acknowledgements H.K. is currently on leave from Sanyo Electric Co., Ltd., Osaka, Japan. The authors thank the Department of Energy and Universal Display Corp. for partial support of this work, as well as R. Holmes and B. W. D'Andrade for discussions.

Author Information Reprints and permissions information is available at npg.nature.com/reprintsandpermissions. The authors declare no competing financial interests. Correspondence and requests for materials should be addressed to S.R.F. (forrest@princeton.edu). 